\title{
Fractal Measures in Market Microstructure Research
}

\author{
Rossitsa Yalamova \\ University of Lethbridge, Canada
}

\begin{abstract}
This paper proposes the generalized use of fractional Brownian motion in a multifractal trading time framework to reveal variation in the index price diffusion process that appears before and after 'extreme' events of distinct origin. "Crashes" following internal self-organization and those caused by external shocks differ in the relaxation process. The goal of this paper is to test for differences in the price diffusion process related to the organization of trading. (JEL: C65, D53 D84, G01, G14)
\end{abstract}

Keywords: trading mechanics, multifractal spectrum, extreme events

\section{Introduction}

Growing competition between different stock exchanges raises the general question of finding the most efficient way to organize securities trading. Financial market microstructure research specifies certain characteristics of a good market: low operational costs, availability of information, liquidity, and information efficiency. Mandelbrot (2001) suggests that price variations do not directly reflect changes in the economic fundamentals, but are rather the outcomes of the structure of the financial system and the agent's responses to a variety of information. Accordingly, this study employs an empirical test of nonlinear volatility modeling (multifractals) in an attempt to reveal information efficiency differences in auction and dealer markets around extreme events.

The major attraction of multifractal processes is their ability to generate long memory in returns of different scales; a process typical of

(Multinational Finance Journal, 2012, vol. 16, no. 1/2, pp. 137-154)

(c) Multinational Finance Society, a nonprofit corporation. All rights reserved.

DOI: $10.17578 / 16-1 / 2-6$ 
financial time-series. This paper proposes a comparison of the multifractal spectra of stock index prices to measure market ability to efficiently process information and to ensure high liquidity. The investigation is based on the Multifractal Model of Asset Returns (MMAR) of Calvet and Fisher (2002). This model assumes that stock prices follow a compound process of Fractional Brownian motion and stochastic trading time capturing the thick tails and long-memory in volatility persistence. Multifractal spectra of stock index prices and trading time may reveal the effect of the trading system on the price function. Pesin and Weiss (1997) motivate the study of multifractals and offer complete multifractal analysis for several classes of dynamical systems.

Johansen, Sornette and Ledoit (2000) assert that financial markets are similar to complex dynamical systems. The different parts of complex systems are linked and affect one another. A complex system may exhibit deterministic and random characteristics with a level of complexity depending on the system's dynamics and its interactions with the environment. But complexity is also related to chaos aperiodic long-term behavior that exhibits sensitive dependence on initial conditions and has limited predictability of subsequent dynamics. The comparative analysis of stock market data during periods of extremes is motivated by the scientific evidence in physics that such complex dynamical systems reveal their properties better under stress than in normal conditions. A better understanding of the dynamics around extreme events would allow the prevention of "crises" or at least achieve more desirable outlier outcomes. Furthermore, in case of chaotic dynamics where small inputs may yield large outcomes, such controls may be feasible at low cost.

Yalamova and McKelvey (2010) suggest that fractality in price dynamics points to herding behavior and self-organization among traders when the market moves away from equilibrium, therefore there is an expectation differences will be detected in the price diffusion process as reflected in different mechanics of trading between auction and dealer markets. Moreover, detecting different self-organization patterns among traders will allow the design of better regulatory and intervention strategies to prevent crashes.

The paper is organized in the following way: market efficiency and trading mechanics for dealer vs. auction markets are discussed in the following section. The article then explains singularities, the Hurst exponent and the multifractal spectrum. The next section introduces 
Fractional Brownian motion in the multifractal trading time model of Calvet and Fisher (2002) and its relevance to research in market microstructure. Presented results are analyzed and suggestions for future research are offered. In brief, the results of the Hurst exponent estimation and the multifractal trading time indicate differences in the index price diffusion process arising from differences in the trading organization. Further research with high frequency data is recommended to reveal finer details.

\section{Dealer vs. Auction Market}

Security exchanges operate as a continuous market with a trading system that can take the form of an auction market or dealer (market maker) market. In an auction market, buy and sell orders are matched according to price and time priority. The order driven trading system works well in a stable market but tends to break down under adverse conditions. The Tokyo Stock exchange does not provide market making services and makes a good example of an auction market for the purposes of this study.

The NASDAQ is a dealer market, wherein market participants are buying from and selling to a market maker, who will always clear trades. This quote driven trading system guarantees immediate execution in the presence of competing market makers. The second example of a dealer market in this study is the London Stock Exchange. Although quote driven until 1997, it differs from the NASDAQ in the first 512 day sample period before the crash of 1987 (starting October 1985). In 1986, the LSE switched from a closed, floor-based, broker-dealer market to an open, electronic quotation system SEAQ, which operates much like the NASDAQ's. In addition to changing its systems, the LSE also enacted new rules designed to encourage competition and narrow quoted spreads. In the second researched period in this study (2001), the LSE operates as a "hybrid" market.

When individuals are buying and selling between one another, an auction takes place, i.e. the highest bidding price will be matched with the lowest asking price. In addition to the auction system, the NYSE has a specialist market system. In case of market imbalance, specialists make adjustments by buying and selling out of their own account, i.e. a "hybrid" market.

In October of 1997, the London Stock Exchange changed its trading 


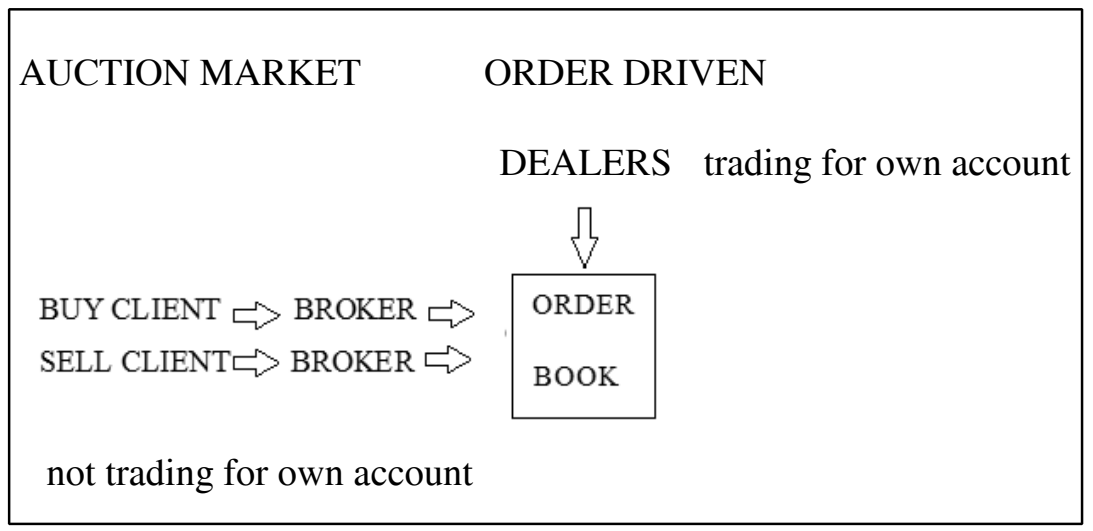

FIGURE 1.- Order flow and execution in an auction market.

system from a dealership system (SEAQ) to an auction system of limit orders (SETS), although choice of trading venue is unconstrained and dealership services are voluntarily supplied.

To compare liquidity between market types, some researchers such as Pagano and Röell (1990) and De Jong, Nijman and Röell (1995) have analyzed the spread and observe that it is lower at the Paris Bourse (auction) than at the LSE (dealer market). Lee (1993) reports lower spreads on the specialist market of the NYSE compared to those at NASDAQ. Similarly, Christie and Huang (1994) find lower trading costs at the NYSE compared to NASDAQ.

In a hybrid market, Friederich and Payne (2002) find that variables which proxy for market-wide liquidity and informational risks also affect the choice of trading venue. This poses the question of whether the coexistence of both trading systems in a market leads to increased efficiency. Efficient market theory suggests that prices adjust quickly to the new information. As new information arrives at the market in a random manner, prices should follow Geometric Brownian motion (in continuous time), where the risk $(\sigma)$ scales with $\sqrt{T}$ which translates in Hurst exponent $H=0.5$ or random walk (in discrete time).

The results show that "hybrid" markets are closest to a random walk $(H=0.5)$, which would support weak-form efficiency. Existing choices of trading venue facilitate quick adjustment of prices to new information. 


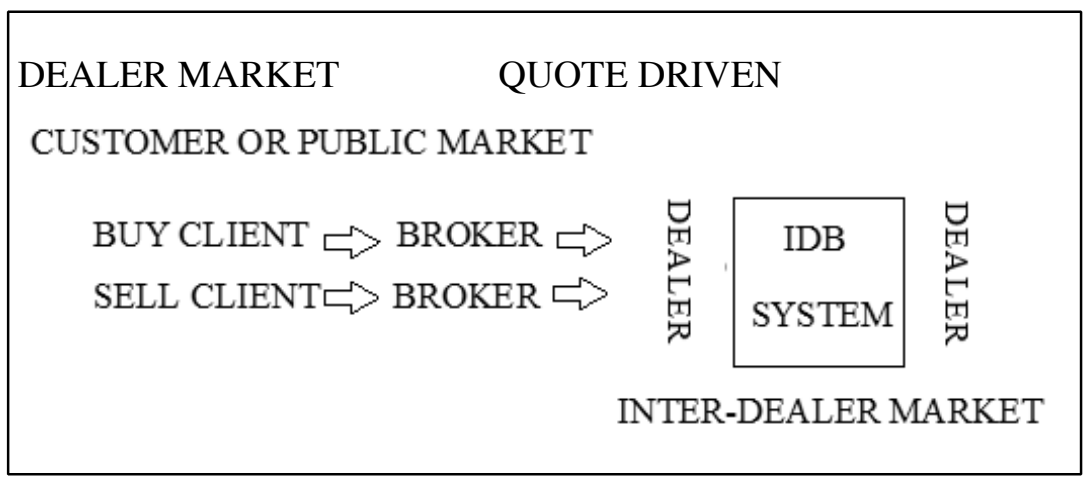

FIGURE 2.- Order flow and execution in a dealer market.

Estimates of the Hurst exponent $0.5<H<1.0$ reveal long memory in the autocorrelation function, i.e. the autocorrelation function $p(\kappa)$ has a very slow decay that follows power law:

$$
p(\kappa)=C \kappa^{-\alpha}
$$

where $C$ is a constant and $\kappa$ is the lag of the autocorrelation function. The Hurst exponent is related to the exponent $\alpha$ of the autocorrelation function:

$$
H=1-\frac{\alpha}{2}
$$

The reported values of the Hurst exponent $(H)$, in either quote driven or order driven markets, point at long memory in the volatility of index prices, which might be caused by inefficient trading organization that slows the process of incorporating news into the stock prices. A Hurst exponent value $(H)$ such that $0.5<H<1$ indicates "persistent behavior" (e.g., a positive autocorrelation).

A number of studies attempt to research the impact of the system on the efficiency of trading. This article's conjecture is that the organization of trading impacts the price diffusion process. Therefore, the scaling function of the index prices is first calculated in order to obtain the Hurst exponent of the Fractional Brownian motion as described by Fisher, Calvet and Mandelbrot (1997). Trading time MFS changes before and after significant drawdowns are compared, attempting to extract information about the development of a "bubble" 
and recovery patterns. Yalamova (2003) also explains the motivation for the research of index price patterns around "market crashes".

\section{Brownian Motion and Multifractals}

\section{A. Hurst Exponent}

The Hurst exponent was first used in hydrology and later in applied mathematics, fractals and chaos theory, and spectral analysis. Benoit Mandelbrot explores the application of the Hurst exponent to financial time series in Fractals and Scaling in Finance (1997). Edgar Peters also discusses the Hurst exponent application to financial time series in Chaos and Order in the Capital markets (1996). The mathematical intuition behind the Hurst exponent is related to the singularity spectrum of a function, i.e. that fractional part $\alpha$ which remains after $n$ time differentiation of the function at point $x_{0}$. Mathematicians also call this the Hölder exponent.

The singularity of a function $g(x)$ is measured by the Hölder exponent $(\alpha)$ at point $x_{0}$ as:

$$
\left|g(x)-P_{x}\left(x-x_{0}\right)\right|<C\left|x-x_{0}\right|^{\alpha L},
$$

where $C$ is a constant and $P_{n}$ is the degree of the polynomial, e. g. $g(x)$ is $n$ times continuously differentiable in $x_{0}$ and the polynomial $P_{n}(x)$ is the first $n+1$ terms of the Taylor series expansion of $g(x)$ in $x_{0}$.

\section{B. Multifractal Spectrum}

Halsey et al. (1986) characterize the singularity of fractal measures by their strength $\alpha$ and their density distribution $f(\alpha)$. The spectrum of singularities is given by the ranges of $\alpha$ and $f(\alpha)$. In monofractal cases the spectrum will collapse to one point only, known as the Hurst exponent $H$. Concavity of the scaling function $\tau(q)$ is evidence of multifractality and existence of more than one singularity exponent. The calculation of the multifractal spectrum of time series is performed on the scaling function $\tau(q)$ via the Legendre transform:

$$
\tau(q) \rightarrow \min _{\alpha}[q \alpha-f(\alpha)]
$$


For more details on the methodology and the algorithm of the multifractal spectrum calculations in Matlab, see Yalamova (2003).

\section{Application to Trading Mechanics}

Gontis and Kaulakys (2004) analyze the relation between the power-law autocorrelation and the origin in the power law probability distribution of trading activity and confirm that the multiplicative stochastic model of the time interval between trades in the financial market is able to reproduce the main statistical properties of trading activity and its power-spectral density. Calvet and Fisher (2002) develop a model of asset returns relating the power spectrum of the price function and the fractality of trading time. Gopikrishnan et. Al. (2000) also provide empirical evidence that the long-range correlations for volatility are due to trading activity.

Therefore, it is suggested that markets with different trading organizations should be tested for differences in the Hurst exponent around market crashes. As argued by Yalamova and McKelvey (2010), self-organization and increased interdependence between traders under conditions of information complexity and ambiguity in the market may lead to bubble build up and changes in the price diffusion process. Better understanding of these dynamics may permit the prudent adaptation of regulations, trading platforms or intervention strategies to prevent or ameliorate market crashes.

\section{Fractional Brownian Motion in Multifractal Trading Time}

Calvet and Fisher (2002) propose a model of asset returns that decomposes the price diffusion process into a long memory process characterized by a Hurst exponent (also called Fractional Brownian motion of the price process) and a time deformation process represented by the multiplicative cascade obtained by a simple iterative procedure. The Itô process is a continuous diffusion that varies as $(d t)^{1 / 2}$ while Fractional Brownian motion has an exponent $0<H<1$, but $H \neq 1 / 2$.On the other side, a multiplicative process has a continuum of values for the exponent whose relative occurrence is summarized in a renormalized probability density - the multifractal spectrum. The multifractal spectrum in the Calvet and Fisher (2002) model is related to the trading 
time deformation process, while the Hurst exponent is calculated from the scaling function $\tau$ and represents the Fractional Brownian motion of the price process.

In order to compare the market characteristics observed before significant "drawdowns" and their after "crash" recovery patterns, the Hurst exponent is estimated from the scaling function $\tau(q)$ according to the following property described in Calvet and Fisher (2002):

$$
\tau\left(\frac{1}{H}\right)=0
$$

\section{A. Hurst Exponent Results}

The Hurst exponents are reported for periods around significant stress situations (October 19, 1987 and September 11, 2001). The assumption is that different causes of instability ("bubble build up" vs. "unexpected event") will also be detectable in the parameters of the price diffusion process.

When the Hurst exponent is greater than 0.5, the price diffusion process exhibits long-memory in volatility. Table 1 illustrates that the persistence in volatility in dealer and auction markets is higher than the persistence in volatility in 'hybrid' markets. Moreover, the persistence in volatility on the London Stock Exchange is much lower in 2001 (after the introduction of SETS) compared to 1987 (dealer system). Increased regularity of the price process before the "crash" of 1987 is also reported in Yalamova (2003) for other international markets, as well as other "bubble build-up" periods. The recovery process from a 'drawdown' is also different in the two types of markets in 1987 and 2001. While the 'hybrid' markets decreased their regularities after a crash following a "bubble", they exhibit much faster recovery in the "unexpected event" case. The "correction" of 1987 is regarded as a natural development of a complex dynamical system that self-organizes, reaches a critical point and returns to equilibrium, while a crash due to unexpected outside event (e.g. September 11, 2001) should not exhibit self-organization and change in the volatility pattern in the preceding period. The assumption of "normal" market before September 11, 2001 is also supported by the estimated average Hurst exponent reported in Yalamova (2003), (i.e. DJIA: 0.5345, S\&P500: 0.5604, FTSE 0.549478). Yalamova and McKelvey (2010) suggest that shock 
TABLE 1. Hurst exponent for the Fractional Brownian motion of daily closing index prices around October 19, 1987 and September 11, 2001.

\begin{tabular}{|c|c|c|c|c|c|}
\hline \multirow[b]{2}{*}{1987} & DJIA & S\&P500 & FTSE & NASDAQ & \multirow{2}{*}{$\frac{\text { NIKKEI }}{\text { auction }}$} \\
\hline & \multicolumn{2}{|c|}{ hybrid } & \multicolumn{2}{|c|}{ dealer } & \\
\hline before & 0.6289 & 0.6180 & 0.6578 & 0.6849 & 0.6849 \\
\hline after & 0.5208 & 0.5464 & 0.5681 & 0.6024 & 0.6097 \\
\hline overall & 0.5952 & 0.5848 & 0.6329 & 0.6329 & 0.6535 \\
\hline 2001 & & hybrid & & dealer & auction \\
\hline before & 0.5208 & 0.5356 & 0.5208 & $\overline{0.6172}$ & 0.6024 \\
\hline after & 0.5780 & 0.5834 & 0.5848 & 0.5917 & 0.5780 \\
\hline overall & 0.5434 & 0.5618 & 0.5649 & 0.6024 & 0.5988 \\
\hline
\end{tabular}

intervention will prevent a self-organizing market from reaching the critical point where a crash will be followed by a slow recovery.

The NASDAQ consistently decreases its regularity in both of these cases of drawdowns, but the change is smaller in the case of external cause for the significant fall in the index. The results for the London Stock Exchange index are comparable to NASDAQ in the first case and similar to DJIA and S\&P500 in the second, which is consistent with the change in the trading system. Moreover, in 1986, the LSE introduced an open electronic quotation system SEAQ, therefore the Hurst exponent was recalculated for the FTSE and NASDAQ for a period of 256 days before the crash of 1987 for better comparison. The Hurst exponent for NASDAQ is $H=0.680272$ for the FTSE is $H=0.696864$ for this period, i.e. volatility persistence is very similar supporting the hypothesized impact of the trading system on index price volatility.

The persistence in volatility in the auction market of the Tokyo Stock exchange index is very similar to that of NASDAQ in both instances. Therefore, the mix of trading systems might produce different patterns in the volatility persistence around stress events, while auction and dealer market display similar patterns within their group. These initial findings lend encouraging support to efforts to expand research in "normal" market and other "stressed" market conditions.

Average Hurst exponents should be viewed with caution as they are only a part of the more complex representation of the asset prices in the Fractional Brownian motion in multifractal trading time framework of Fisher, Calvet and Mandelbrot (1997). Fractional Brownian motion is too homogeneous for the representation of financial asset price 


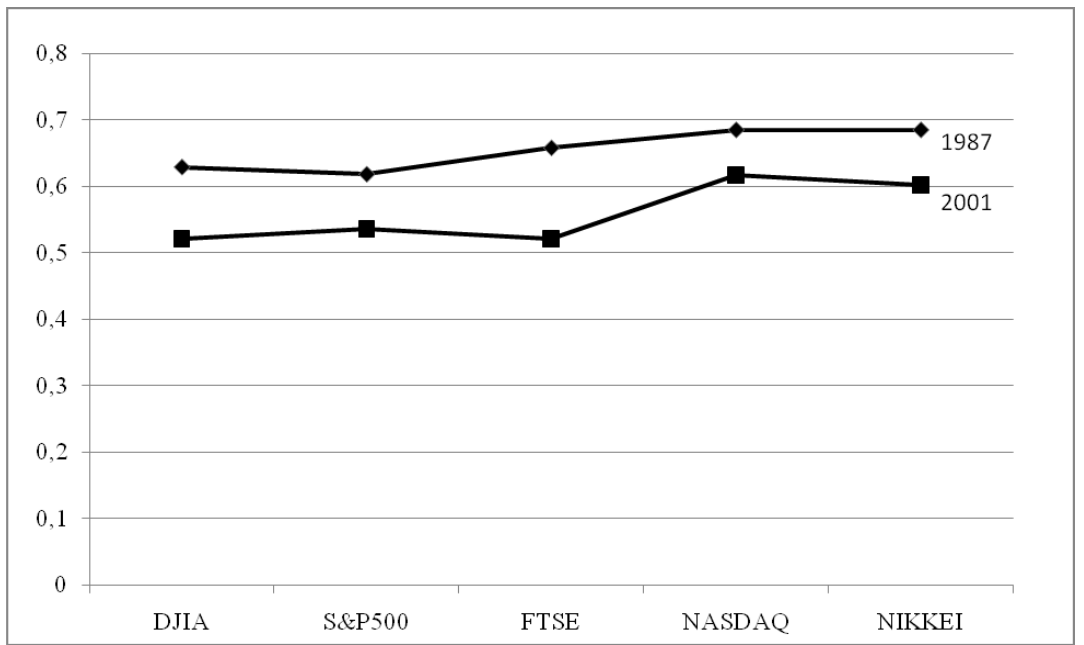

FIGURE 3.- The Hurst exponent in the 'hybrid' markets is lower before "drawdowns". Assuming that before September 11, 2001 markets were around 'normal', a process close to a random walk was observed for DJIA, S\&P500 and FTSE (e.g. "hybrid" markets are more efficient).

processes, as the irregularity exponent should be the same at all times. If the $H$ exponent varies with time, the process exhibits multifractal structure. A test of the multifractality of a process involves the linearity of the scaling function $\tau(q)$.

The daily index price time series exhibits deviations from linearity of the scaling exponent $\tau(q)$ suggesting the existence of a multifractal spectrum, instead of one monofractal Hurst exponent. In the Finance literature, Corrazza and Malliaris (2002) draw a similar conclusion for a number of foreign currency markets. Muzy, Delour and Bacry (2000) use the non-linearity of the scaling function as evidence of multifractality of price fluctuations of financial time-series. Schmitt, Schertzer and Lovejoy (2000), Bacry, Delour and Muzy (2001), and Turiel and Pérez-Vincente (2002) use the non-linearity of the scaling function to show that the estimate of two moments is by no means sufficient for describing the entire distribution, proposing the use of multifractal analyses and models in Finance research. Among the important implications of multifractal analysis are the characterization of all order moments and the validation of scaling models. 


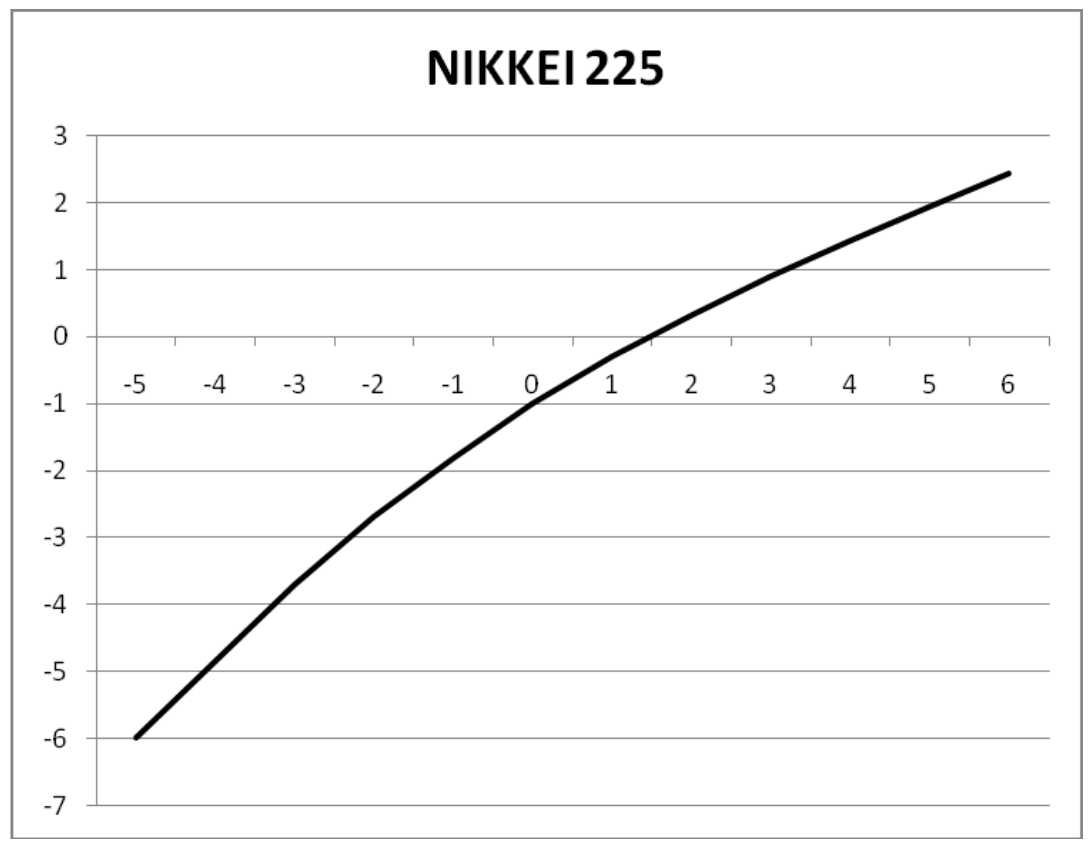

FIGURE 4.- The scaling functions $\tau(q)$ of the index price series exhibit concavity. (on the y-axis: the scaling exponent, on the x-axis: $q$-th moment).

\section{B. Multifractal Trading Times Results}

A fractional Brownian motion with Hurst exponent $(H)$ between 0 and 1 is defined by a stationary increment process that follows a normal distribution with zero mean and variance $\sigma^{2 H}$. Self-similar stable processes are obtained when a stable distribution replaces the normal. Such processes have discontinuous paths with fractal properties, well adapted for infinite variance stochastic modeling.

Monofractal Brownian motion and self-similar stable processes often lack the flexibility to model real-world situations due to their simplicity in scaling or due to non-stationarity of the underlying process. Multifractal processes with non-linear scaling function and stationary zero mean increments are proposed by Riedi (2002). This implies a multifractal 'time warp', i.e., an increasing function or process $M(t)$ for which the multifractal formalism holds and a process $B$ with monofractal scaling properties $H$ (fractional Brownian Motion) form the compound process $\beta(t)$ : 


$$
\beta(t) \triangleq B_{H}(M(t))
$$

In this case, $M(t)$, stands for a monotonic multiplicative cascade with flexible multifractal properties and $B_{H}$ generally represents any self-similar process with index $H$. The compound process $\beta(t)$ combines the rich multifractal structure of a multiplicative cascade along with the self-similarity and the non-monotonicity of Fractional Brownian motion paths. Equality in respect to probability distribution holds almost everywhere (a. e.) or almost surely (a. s.), in the sense of finite dimensional distribution.

A cascade model accounts for the distribution of the volatility of returns across scales but not for the fluctuation of these returns. The multiplicative cascade model predicts strong correlations in the volatility, while the truncated Lévy model (previously considered for financial time series) assumes no such correlations. Ghashgaie et al. (1996) propose a multiplicative cascade model for exchange rate fluctuation. Arneodo, Muzy and Sornette (1998) reveal a multiplicative cascade process in the volatility of the S\&P500 return data.

The rigid correlation structure of Fractional Brownian motion is somewhat restrictive for modeling purposes. Also, the slow decay of its auto-correlation function inspires a weaker notion of "similarity on all scales" in terms of second-order statistics, defined on varying lags of the increment process rather than on the process itself.

Statistically self-similar and monofractal or multiplicative processes may be defined on one side and almost surely increasing and multifractal processes on the other side such that a multiplicative cascade leads to multifractality and a strong deviation from normality. Calvet and Fisher (2002) introduce a multifractal model of asset returns that compounds a Brownian motion with a multifractal time deformation process. Gaussianity, long range dependence and multifractal structure are characteristics of Brownian motion in multifractal time. If $B_{H}(t)$ is a fractional Brownian motion and stochastic trading time $\theta(t)$ is a multiplicative process, then the compound process $X(t):=B_{H}[\theta(t)]$ is fractional Brownian motion in multifractal trading time, combining a long range dependence process and positive increment process with an underlying multifractal structure.

The multifractal spectrum reflects the n-point correlations and thus provides more information about the temporal organization of price fluctuations than 2-point correlations. The multifractal spectrum $f(\alpha)$ is a convex and single-humped function with a maximum at $\alpha_{0}$, where 
TABLE 2. The multifractal spectrum of price series with capacity dimension at $\alpha_{0}$ and very irregular instances at $\alpha_{\text {min }}$.

\begin{tabular}{|c|c|c|c|c|c|c|}
\hline \multirow[b]{2}{*}{1987} & \multirow[b]{2}{*}{$\operatorname{MFS} \alpha$} & DJIA & S\&P500 & FTSE & NASDAQ & \multirow{2}{*}{$\frac{\text { NIKKEI }}{\text { auction }}$} \\
\hline & & \multicolumn{2}{|c|}{ hybrid } & \multicolumn{2}{|c|}{ dealer } & \\
\hline \multirow[t]{2}{*}{ before } & $\alpha_{0}$ & 0.5201 & 0.4911 & 0.5454 & 0.5506 & 0.5863 \\
\hline & $\alpha_{\min }$ & 0.2424 & 0.1987 & 0.1728 & 0.2625 & 0.3203 \\
\hline \multirow[t]{2}{*}{ after } & $\alpha_{0}$ & 0.3966 & 0.4184 & 0.4077 & 0.4898 & 0.5236 \\
\hline & $\alpha_{\min }$ & 0.0886 & 0.1193 & 0.1478 & 0.1134 & 0.0436 \\
\hline 2001 & & & hybrid & & dealer & auction \\
\hline \multirow[t]{2}{*}{ before } & $\alpha_{0}$ & 0.2928 & 0.3449 & 0.3051 & 0.4610 & 0.4323 \\
\hline & $\alpha_{\min }$ & 0.1228 & 0.1697 & 0.2252 & 0.2469 & 0.2222 \\
\hline \multirow[t]{2}{*}{ after } & $\alpha_{0}$ & 0.3890 & 0.4081 & 0.3964 & 0.4172 & 0.4159 \\
\hline & $\alpha_{\min }$ & 0.2568 & 0.2940 & 0.2551 & 0.3142 & 0.2578 \\
\hline
\end{tabular}

$f\left(\alpha_{0}\right)$ is also called the capacity dimension $D_{0}$. Scaling of positive moments is estimated by $\alpha$ 's with values lower than $\alpha_{0}$. The scaling of the highest moment given by $\alpha_{\min }$ in the empirical measurements reaches down to values much lower than 0.5 , representing an irregular price process with high risk for investors.

The Tokyo Stock Exchange exhibits high capacity dimensions $\alpha_{0}$ and comparatively high $\alpha_{\min }$ (low risk) for the price process before the crash of 1987, while the two dealer markets follow the hybrid markets, although with lower capacity dimensions that are not much riskier than the dealer markets as measured by $\alpha_{\text {min }}$. The multifractal spectra (MFS) before the crash of 1987 are different from the respective MFS in 2001. Markets exhibit higher regularity before the crash of 1987 that is in line with the hypothesis of "pressure build-up" reported in Yalamova (2003). The multifractal spectrum of the price process is an intermediate step that allows one to calculate the multifractal trading time spectrum. According to the MMAR model of Fisher, Calvet and Mandelbrot (1997), prices follow a compound process of fractional Brownian motion and multifractal trading $\theta$. The multifractal spectra calculated from the price function defines the multifractal spectrum of the trading time $\theta$ as:

$$
f_{p}(\alpha)=f_{\theta}\left(\frac{\alpha}{H}\right)
$$

The multifractal trading time $\mathrm{a}_{0}$ at the Tokyo Stock Exchange remains the same after the event date. It is also observed that risk increases 
TABLE 3. The multifractal spectrum of trading time with swift passage of time at low $\alpha_{\text {min }}$.

\begin{tabular}{|c|c|c|c|c|c|c|}
\hline \multirow[b]{2}{*}{1987} & \multirow[b]{2}{*}{ MFS $\alpha$} & DJIA & S\&P500 & FTSE & NASDAQ & \multirow{2}{*}{$\frac{\text { NIKKEI }}{\text { auction }}$} \\
\hline & & \multicolumn{2}{|c|}{ hybrid } & \multicolumn{2}{|c|}{ dealer } & \\
\hline \multirow[t]{2}{*}{ before } & $\alpha_{0}$ & 0.8269 & 0.7947 & 0.7827 & 0.8038 & 0.8559 \\
\hline & $\alpha_{\min }$ & 0.3855 & 0.3214 & 0.2479 & 0.3832 & 0.4677 \\
\hline \multirow[t]{2}{*}{ after } & $\alpha_{0}$ & 0.7616 & 0.7656 & 0.7192 & 0.8130 & 0.8588 \\
\hline & $\alpha_{\min }$ & 0.1701 & 0.2183 & 0.2606 & 0.1882 & 0.0716 \\
\hline 2001 & & & hybrid & & dealer & auction \\
\hline \multirow[t]{2}{*}{$\overline{\text { before }}$} & $\alpha_{0}$ & 0.5636 & 0.6439 & 0.5857 & 0.7469 & 0.7176 \\
\hline & $\alpha_{\min }$ & 0.2364 & 0.3168 & 0.4233 & 0.4001 & 0.3688 \\
\hline \multirow[t]{2}{*}{ after } & $\alpha_{0}$ & 0.6730 & 0.6994 & 0.6778 & 0.7051 & 0.7195 \\
\hline & $\alpha_{\min }$ & 0.4444 & 0.5039 & 0.4362 & 0.5309 & 0.4459 \\
\hline
\end{tabular}

significantly in the "after crash" trading of 1987, exhibiting the highest risk of all markets with outbursts of fast trading that may indicate system breakdown in adverse conditions. In its information efficiency aspect, the September 11, 2001 event might have been quickly discounted. Markets, (except for FTSE), exhibit a decrease in risk measured by $\alpha_{\min }$, however the concavity of the spectrum for lower Hölder exponents (more irregular instants) implies disproportionate contributions to volatility. The measure of trading time per unit of clock time is high for low $\alpha$ 's and is related to swift passage of trading time according to Fisher et al. (1997). As explained in this model, the price process has one Hurst exponent; therefore the irregularities with low $\alpha$ 's are due to trading time.

This model accommodates a wide range of financial prices and permits the identification of a multiplicative measure empirically from the estimated spectra. The daily data multifractal spectra are quadratic, generated by $\log$ normally distributed multipliers $M\left(-\log _{b} M \sim N\left(\lambda, \sigma^{2}\right)\right.$. Calvet and Fisher (2002) derive the calculation of $\lambda$ from the multifractal spectrum of trading time $\left(\lambda=\alpha_{0} / H\right)$. The dealer markets were much faster in trading around the crash of 1987. While all markets exhibit a pattern of slowing down after the crash, the results suggest that the hybrid markets exhibit the most pronounced slowdown. The results for the period of 2001 are mixed. Inferences on the volatility pattern connection to the trading systems cannot be made yet on such a small sample. Also, the S\&P500 index includes 500 stocks traded on different exchanges, while the DJIA includes 30 stocks traded on NYSE and 
TABLE 4. Trading time multifractal spectrum is characterized by lognormally distributed multipliers $M\left(-\log b M \sim N\left(\lambda, \sigma^{2}\right)\right), \lambda$ 's are reported in the table.

\begin{tabular}{|c|c|c|c|c|c|c|}
\hline \multirow[b]{2}{*}{1987} & \multirow[b]{2}{*}{$\operatorname{MFS} \lambda$} & DJIA & S\&P500 & FTSE & NASDAQ & \multirow{2}{*}{$\frac{\text { NIKKEI }}{\text { auction }}$} \\
\hline & & \multicolumn{2}{|c|}{ hybrid } & \multicolumn{2}{|c|}{ dealer } & \\
\hline$\overline{\text { before }}$ & $\lambda$ & 1.3148 & 1.2977 & 1.1232 & 1.1736 & 1.2497 \\
\hline after & $\lambda$ & 1.4566 & 1.4011 & 1.2687 & 1.3496 & 1.4084 \\
\hline 2001 & & & hybrid & & dealer & auction \\
\hline$\overline{\text { before }}$ & $\lambda$ & 1.0849 & 1.2023 & 1.1246 & $\overline{1.2099}$ & 1.1978 \\
\hline after & $\lambda$ & 1.1644 & 1.1988 & 1.1590 & 1.1916 & 1.2448 \\
\hline
\end{tabular}

NASDAQ. Therefore, while it can be recognized that these are not close proxies for hybrid markets, they could yet be seen as examples illustrating the power of a new advanced methodology for volatility and non-linear dynamics research.

\section{Conclusions}

The goal of market microstructure research under the efficient market hypothesis (EMH) is typically to define trading mechanisms and regulations that will lead to information efficiency and transparency such that new information is quickly and reliably incorporated into stock prices. Traders are portrayed as independent rational decision makers and, as information arrives to the market in random fashion, prices follow Brownian motion and no long memory in the autocorrelation function of returns is observed. Although the EMH allows for some temporary deviations (anomalies), they are classified as random and therefore do not permit arbitrage.

Multifractality in asset returns has been empirically confirmed in stock and foreign currency markets and has found its way into the Multinational Finance Journal in Corraza and Malliaris (2002). Acceptance of fractality though, leads to a number of problems in mathematical modeling and derivatives pricing including the loss of martingale properties. As argued extensively in Yalamova and McKelvey (2010), "Fractal Finance" should not be seen as a competing alternative to existing research within the efficient market paradigm, but as an extension to accommodate situations having high information 
complexity and trader interactions coupled with positive feedback. In such "bubble build-up" regimes, trader interaction feeds back to influence individual decisions and the market self-organizes towards the critical point.

The difference between a crash as a result of an unexpected event (e.g. September 11, 2001) and as a result of self-organization of trading activities and bubble burst should show up in the price diffusion process before and after the event. The multifractal spectra of stock index price time series were analyzed in light of the Multifractal Model of Asset Returns of Calvet and Fisher (2002). A multifractal formalism based on wavelet transform modulus maxima (WTMM), introduced by Muzy, Bacry and Arneodo (1993), permits the determination of the whole singularity spectrum $f(\alpha)$ directly from any experimental time-series. This works in most situations and provides a unified multifractal description of self-affine distributions. Additional technical details, the Matlab algorithm, and an intuitive explanation of wavelet (time/frequency) decomposition of financial time series are available by request.

Data on daily closing prices for DJIA, S\&P500, FTSE, NASDAQ and NIKKEI 225 indices were analyzed around the crash of 1987 and September 11, 2001 since complex dynamical systems reveal their structure and properties better under stress (i.e. in extreme conditions). The irregularity exponents and the Hausdorff dimensions of the multifractal spectra were analyzed for differences in the volatility and trading patterns. The goal of this paper is to increase interest in research using multifractality to better describe the behavior of financial markets. Although non-linearity is detected and characterized with parameters of the multifractal trading time framework, more empirical evidence is needed. The appropriateness of the proposed tests for market microstructure research is advocated. Multifractal Model of Asset Returns framework reveals information about the 'regularity' build up before the crash of 1987 and the recovery period volatility and trading time changes. Further research should be performed on high frequency data.

Fractional Brownian motion with a compound trading time deformation process represents an appropriate framework for market microstructure research. The multifractal spectrum of trading time permits the recovery of the multipliers of the underlying multiplicative cascade process. This multifractal process reveals the interaction between traders leading to information cascade and herding. A dynamic 
model of trading behavior should help policy makers to chose trading organization structures, regulations, and crisis intervention strategies that will both improve efficiency and prevent or ameliorate crashes.

Multifractal decomposition of invariant sets with complicated geometry is an important part of the research of complex dynamical systems such as financial markets. The multifractal spectrum (MFS) reflects the n-point correlations and thus provides more information about the temporal organization of price fluctuations than 2-point correlations. MFS also can be used for the characterization of strange attractors for chaotic dynamical systems (Halsey et al. 1986), a topic of interest among Finance researchers but lacking consistent methods and sufficient empirical evidence.

Accepted by: Prof. P. Theodossiou, Editor-in-Chief, June 2011

\section{References}

Arneodo, A.; Muzy, J.-F.; and Sornette, D. 1998. "Direct" casual cascade in the stock market. The European Physical Journal B 2; 277-282.

Bacry, E.; Delour, J.; and Muzy, J. 2001. A Multifractal Random Walk. Physical review E 64; 026103-026106.

Calvet, L., and Fisher, A. 2002. Multifractality in Asset Returns: Theory and Evidence. The Review of Economics and Statistics 84; 381-406.

Corazza, M., and Malliaris, A. G. 2002. Multi-fractality in foreign currency markets. Multinational. Finance Journal 6; 65-98.

Christie, W., and Huang, R. 1994. Market Structure and Liquidity: A Transaction Data Study of Exchange Listings. Journal of Financial Intermediation 3; 300-326.

De Jong, F.; Nijman, T.; and Roell, A. 1995. A Comparison of the cost of trading French shares on the Paris Bourse and on SEAQ. International European Economic Review 39; 1277-1301.

Fisher, A.; Calvet, L.; and Mandelbrot, B. 1997. Multifractality of Deutschemark/US Dollar Exchange Rates. Cowles Foundation Discussion Paper \# 1165.

Friederich, S., and Payne, R. 2002. Dealer Liquidity in an Auction Market: Evidence from the London Stock Exchange. Working paper. http://ssrn.com/abstract=348080.

Ghashgaie, S.; Breymann, W.; Peinke, J.; Talkner, P.; and Dodge, Y. 1996: Turbulent cascades in foreign exchange markets. Nature 381; 767-770.

Gontis, V., and Kaulakys, B. 2004. Modeling Financial Markets by the Multiplicative Sequence of Trades. Physica A 344; 128-133. 
Gopikrishnan, P.; Plerou, V.; Liua, Y.; Amaral, L.A.N.; Gabaix, X.; and Stanley, H. E. 2000. Scaling and correlation in financial time series. Physica A 287; 362-373.

Halsey, T. C.; Jensen, M.H.; Kadanoff, L.P.; Procaccia, I.; and Shraiman, B. I. 1986. Fractal measures and their singularities: The characterization of strange sets. Physical. Review A 33; 1141-1151.

Johansen, A.; Sornette, D.; and Ledoit, O. 2000. Crashes as Critical Points. International Journal of Theoretical and Applied Finance 3(2); 219-255.

Lee, C. 1993. Market Integration and Price Execution for NYSE-Lsted Securities. Journal of Finance 48; 1009-1038.

Mandelbrot, B. B. 1997. Fractals and Scaling in Finance, Discontinuity, Concentration, Risk. Springer, New York.

Mandelbrot, B. 2001. Scaling in financial prices: III. Cartoon Brownian motions in multifractal time. Quantitative Finance 1(4); 427-440.

Muzy, J. F.; Bacry, E.; and Arneodo, A. 1993. Multifractal Formalism for Fractal Signals: The Structure Function Approach versus the Wavelet-Transform Modulus-Maxima Method. Physical Review E 47(2); 875-884.

Muzy, J.; Delour, J.; and Bacry, E. 2000. Modeling Fluctuations of Financial Time Series: from Cascade Process to Stochastic Volatility Model. European Physics Journal B 17; pp. 537-548.

Pagano, M., and Roell, A. 1990. Trading Systems in European Stock Exchanges: Current Performance and Policy Options. Economic Policy 10; 63-115.

Pesin, Y., and Weiss, H. 1997. The Multifractal Analysis of Gibbs Measures: Motivation, Mathematical Foundation, and Examples. Chaos 7(1); 89-106.

Peters, E. E. 1996. Chaos and Order in the Capital Markets: A New View of Cycles, Prices, and Market Volatility. Wiley Finance.

Riedi, R. H. 2002. Multifractal Processes. In Doukhan, Oppenheim and Taqqu (eds). Long range dependence : theory and applications. Birkhäuser Publishing; 615-725.

Schmitt, F.; Schertzer, D.; and Lovejoy, S. 2000. Multifractal Fluctuations in Finance. International Journal of Theoretical and Applied Finance 3; 361-364.

Turiel, A., and Pérez-Vicente, C. J. 2003. Multifractal Geometry in Stock Market Time-Series. Physica A 322; 629-649.

Yalamova, R. 2003. Wavelet MRA of Index Patterns around Financial Market Shocks. PhD thesis. Kent State University.

Yalamova, R., and McKelvey, B. 2010. Explaining What Leads Up to Stock Market Crashes: A Phase Transition Model and Scalability Dynamics. The Journal of Behavioral Finance (in press). 